DOI: https://doi.org/10.11144/Javeriana.upsy17-5.vifm

\title{
Validez e invarianza factorial de una medida breve de Satisfacción con la Vida Familiar*
}

Validity and Factorial Invariance of a Brief Measure of Satisfaction with Family Life

Recepción: 18 Mayo 2018 | Aceptación: 06 Junio 2018

\section{TOMÁs CAYCHO-RODRÍGUEZ ${ }^{\text {a }}$}

Universidad Privada del Norte, Perú

ORCID: http://orcid.org/0000-0002-5349-7570

José Ventura-LeÓN

Universidad Privada del Norte, Perú

ORCID: http://orcid.org/0000-0003-2996-4244

Miguel Barboza-Palomino

Universidad Privada del Norte, Perú

ORCID: http://orcid.org/0000-0001-8045-5491

Mario Reyes-Bossio

Universidad Peruana de Ciencias Aplicadas, Perú

ORCID: http://orcid.org/0000-0003-4655-1927

Walter L. Arias Gallegos

Universidad Católica San Pablo, Perú

ORCID: http://orcid.org/0000-0002-4183-5093

Cirilo H. García Cadena

Universidad Autónoma de Nuevo León, México

ORCID: http://orcid.org/0000-0001-6066-7745

IsABEl Cabrera-Orosco

Universidad Continental, Perú, Perú

ORCID: http://orcid.org/0000-0002-0375-2879

Jonathan Ayala

Universidad del Cono Sur de las Américas, Paraguay

ORCID: http://orcid.org/0000-0002-3651-3356

Katherine Morgado-Gallardo

Universidad Católica del Maule, Chile

ORCID: http://orcid.org/0000-0002-6923-2534

Julio Cesar Huamani Cahua

Universidad Católica San Pablo, Perú

ORCID: http://orcid.org/0000-0001-8159-803X

a Autor de correspondencia. Correo electrónico:

tomas.caycho@upn.pe

Para citar este artículo: Caycho-Rodríguez, T., VenturaLeón, J., Barboza-Palomino, M., Reyes-Bossio, M., Arias Gallegos, W. L., García Cadena, C. H., ... Huamani Cahua, J. C. (2018). Validez e invarianza factorial por sexo de una medida breve de Satisfacción con la Vida Familiar en escolares de Lima (Perú). Universitas Psychologica, 17(5), 1.17. https://doi.org/ 10.11144/Javeriana.upsy17-5.vifm

\section{RESUMEN}

Se examinó la estructura factorial, la confiabilidad, la validez convergente y discriminante, y la invarianza factorial por sexo, de la Satisfaction with Family Life Scale (SWFLS). Los participantes fueron 804 estudiantes de primaria y secundaria de escuelas privadas de Lima, con un promedio de edad de 13.5 años $(\mathrm{DE}=1.6)$. Los resultados confirman la estructura unidimensional de la escala $\left(\chi^{2}=3.49, d f=5, p=0.63 ; \chi^{2} / d f=0.69\right.$; GFI $=0.998 ; \mathrm{CFI}=1$; NFI $=0.998 ; \mathrm{RMSEA}=0[\mathrm{IC} 90 \% 0,0.038]$ y SRMR $=0.007)$, la invarianza factorial por sexo y una consistencia interna adecuada $(\alpha=0.84 ; \omega=0.84 ; H=0.85)$. Se obtuvieron evidencias 
satisfactorias de validez convergente y discriminante de las SWFLS con medidas de funcionalidad familiar, bienestar subjetivo y depresión. En conclusión, la SWFLS presenta excelentes propiedades psicométricas para medir la satisfacción con la vida familiar en escolares limeños.

Palabras clave

análisis factorial confirmatorio; invarianza factorial; satisfacción con la vida familiar; escolares peruanos.

\begin{abstract}
We examined the factor structure, reliability, convergent and discriminant validity, and factorial invariance by sex of the Satisfaction with Family Life Scale (SWFLS). The participants were 804 primary and secondary students from private schools in Lima, with an average age of 13.5 years $(\mathrm{SD}=1.6)$. The results confirm the one-dimensional structure of the scale $\left(\chi^{2}=3.49, d f=5, p=0.63, \chi^{2} / d f\right.$ $=0.69$, GFI $=0.998, \mathrm{CFI}=1, \mathrm{NFI}=0.998$, RMSEA $=0$ [IC90\% 0, 0.038], and SRMR $=0.007$ ), factorial invariance by sex and adequate internal consistency $(\alpha$ $=0.84 ; \omega=0.84 ; \mathrm{H}=0.85)$. Satisfactory evidence of convergent and discriminant validity of the SWFLS was obtained with measures of family functionality, subjective well-being and depression. In conclusion, the SWFLS presents excellent psychometric properties to measure satisfaction with family life in Lima schoolchildren.

Keywords

confirmatory factor analysis; factorial invariance; satisfaction with family life; Peruvian schoolchildren.
\end{abstract}

En los últimos años, se ha incrementado el interés por la investigación de los juicios que niños y adolescentes en edad escolar tienen acerca de la satisfacción con la vida (SWL, por sus siglas en inglés) (Suldo, Savage, \& Mercer, 2014) y su importancia para el buen funcionamiento psicológico, educativo, social y físico (Suldo, Huebner, Friedrich, \& Gilman, 2009), presentando implicaciones importantes para la práctica psicológica a nivel escolar (Gilman \& Huebner, 2003; Huebner, 2004). La SWL es la dimensión cognitiva y el indicador más estable del bienestar subjetivo (Emerson, Guhn, \& Gadermann, 2017), que se define como la evaluación que se realiza de la vida en general (Diener \& Ryan, 2009). Así, la investigación acerca de la SWL permite una visión más completa acerca de la salud mental de los adolescentes en edad escolar que no se limita a la identificación de los síntomas psicopatológicos (Gilman \& Huebner, 2003).
En este sentido, las medidas de SWL, a diferencia de otras medidas psicológicas positivas y psicopatológicas en adolescentes, demuestran los beneficios de medir comportamientos positivos y negativos en las evaluaciones del bienestar en esta población (Antaramian, Huebner, Hills, \& Valois, 2010; Suldo \& Shaffer, 2008). En muestras de adolescentes en edad escolar, elevados niveles de SWL y ausencia o presencia mínima de psicopatología están relacionados con mejores logros educativos, adecuado establecimiento de relaciones sociales y una mejor salud física, en comparación con otros adolescentes que informaron bajos niveles de satisfacción con la vida (Suldo \& Shaffer, 2008). Asimismo, una alta SWL permite actitudes más favorables hacia los docentes y la escuela, así como un mayor compromiso cognitivo y aspiraciones académicas (Gilman \& Huebner, 2006; Lewis, Huebner, Malone, \& Valois, 2011; Proctor, Linley, \& Maltby, 2010). Con respecto a la actividad físico-deportiva, favorece el desarrollo de mejores hábitos de salud, autoconcepto y motivación (González-Serrano, Huéscar, \& Moreno-Murcia, 2013).

Un estudio longitudinal de cuatro años en adolescentes de Hong Kong, muestra que la SWL funciona como un mediador en la relación entre el rendimiento académico percibido y la desesperanza (Shek \& Li, 2016). De igual manera, adolescentes con mayores niveles de SWL son menos vulnerables a los efectos del desempleo familiar (Frasquilho, Matos, Neville, Gaspar, \& Almeida, 2016), están asociados a mayores niveles de mindfullness y autoevaluaciones nucleares positivas (Jianfeng, Wu, Hongwei, \& Yulan, 2016), decrece la probabilidad de comportamientos riesgosos (Çakar, Tagay, \& Karataş, 2015) y la categorización como víctimas de bullying (Totan, Özer, \& Özmen, 2017).

La SWL hace también referencia a la evaluación global de los dominios específicos de la vida como el trabajo, la familia, los amigos, el deporte, la actividad fisica, entre otros (Balaguer, Castillo, \& Duda, 2008; Huebner, Suldo, \& Gilman, 2006). Dentro de los dominios específicos, en los últimos años, la satisfacción 
con la vida familiar (SWFL) definida como el grado en que las personas se consideran satisfechas con su vida familiar (Luna \& Laca, 2014), ha sido considerada como un área de estudio importante debido a su relación con indicadores de salud, calidad de vida, estrategias de afrontamiento al estrés, cohesión familiar, adaptabilidad, comunicación y funcionamiento familiar en general (Guillén et al., 2011; Poff, Zabriskie, \& Townsend, 2010). Diversos estudios (Easterlin, 2006; Moss \& Willoughby, 2016) reportan que las personas más felices y con una mayor SWL son aquellas que se encuentran más satisfechas con su vida familiar, mientras que otros hallazgos (Moss \& Willoughby, 2016) señalan que mayores niveles de SWFL se encuentran relacionados de forma positiva con la satisfacción en los ámbitos financiero, comunitario y laboral.

El estudio de la SWFL en adolescentes resulta aún más importante si se considera que para este grupo, la familia es una de las principales fuentes de apoyo, seguridad, protección y resiliencia (Arnett, 2008; Barboza-Palomino et al., 2017; González, Valdez, \& Zavala, 2008) por encima de lo ofrecido por la pareja, la escuela o la religión (Serrano \& Flores, 2004); aunque su influencia suele ser más fuerte durante la infancia y la niñez que durante la adolescencia y la juventud (Arias, Quispe, \& Ceballos, 2016). Además, la evidencia reporta que en muestras de adolescentes en edad escolar, mayores niveles de satisfacción familiar favorecen la presencia de afectos positivos (éxito, hedonismo emocional, esperanza, entre otros), así como la evaluación afectivo-cognitiva de la vida en general y la satisfacción con dominios específicos de la vida (dinero, amigos, trabajo, salud, escuela), mientras que correlaciona negativamente con los afectos negativos (expresividad negativa, emocionalidad negativa, nerviosismo, etc.) (Luna, Laca, \& Mejía, 2011). Con base en lo anterior, se requiere disponer de instrumentos de medida confiables, válidos y útiles para la medición de la SWFL (Tasdelen-Karçkay, 2016), ya que una medida efectiva proporcionará el marco necesario para la identificación de factores comportamentales que promuevan una adecuada calidad de vida familiar (Zabriskie \& Ward, 2013).

La teorización y medición de la satisfacción familiar se inicia a mediados de 1970 (Barraca, López, \& Olea, 2000; Tasdelen-Karçkay, 2016; Zabriskie \& Ward, 2013) con los trabajos iniciales de Campbell, Converse y Rodgers (1976) y de Andrews y Withey (1976). Desde entonces, los estudios acerca de la satisfacción familiar se han realizado en poblaciones específicas, así como en diferentes grupos de edad (TasdelenKarçkay, 2016). En este contexto, se considera al Family Life Questionnaire ([FLQ]; Guerney, 1977) como el instrumento pionero para la medida de la satisfacción con la vida familiar (Barraca et al., 2000).

A inicios de la década de 1980, surgen nuevos modelos para la medición de la satisfacción familiar, como el circunflejo de Olson de 1978, del cual se deriva la muy conocida Escala de Evaluación de Adaptabilidad y Cohesión Familiar (FACES, por sus siglas en inglés) que desde su versión original FACES I (Olson, Bell, $\&$ Portner, 1978), ha tenidos diferentes versiones (Bazo-Alvarez et al., 2016). Adicionalmente, bajo este modelo teórico, se construye la Escala de Satisfacción Familiar ([ESF]; Olson \& Wilson, 1982), conformada por 14 ítems que se convirtió en una de las medidas de satisfacción familiar más utilizadas (Zabriskie \& Ward, 2013) y que en la actualidad tiene una versión reducida de 10 ítems, la cual mide la satisfacción acerca del funcionamiento, cohesión, flexibilidad y comunicación familiar (Olson \& Wilson, 1982).

Además, existen otras escalas de medida como la Kansas Family Life Satisfaction Questionnaire ([KFLSQ]; McCollum, Schumm, \& Russell, 1988; Schumm, McCollum, Bugaighis, Jurich, \& Bollman, 1986), la Family Satisfaction Scale ([FSS]; Carver \& Jones, 1992) y más recientemente, la Family Life Satisfaction Scale ([FLSS]; Barraca et al., 2000). La mayoría de los instrumentos anteriores, tales como el FACES, ESF, FSS y FLSS, se caracterizan por tener entre 10 y 27 ítems, mientras que otros, como el KFLSQ, son más breves, pero con limitada evidencia de validez y confiabilidad, además, su ámbito de aplicación son familias con cuatro 
o más miembros, incluido un matrimonio y al menos dos hijos (Schumm et al., 1986).

Ante esto, se requieren instrumentos con evidencias de validez y confiabilidad, de rápida y fácil aplicación a diversos grupos poblacionales, entre ellos los adolescentes, y que a su vez generen una buena aceptación de parte de los profesionales de los servicios educativos y que sean tolerados y aceptados por los adolescentes. Entre estos, se encuentra la Satisfaction With Family Life Scale (SWFLS) (Zabriskie \& McCormick, 2003; Zabriskie \& Ward, 2013) que es una medida breve de la SWFL conformada por cinco ítems. La SWFLS es una versión modificada de la Satisfaction With Life Scale ([SWLS]; Diener, Emmons, Larsen, \& Griffin, 1985), donde en cada uno de los cinco ítems originales la palabra "vida" fue reemplazada por la expresión "vida familiar". A pesar de la existencia de críticas frente al enfoque global de la satisfacción con la vida (Strack, 1999), esta escala presenta solidez, es recomendada y apropiada en el contexto familiar (Zabriskie \& Ward, 2013). De esta manera, la SWFLS permite evaluar diversos dominios familiares en relación con su propia estructura de valores, experiencias y expectativas, llegando a un juicio global de satisfacción familiar (Zabriskie \& Ward, 2013).

La SWFLS, creada originalmente para usarse en padres e hijos adolescentes estadounidenses entre 11 y 15 años, se ha aplicado a personas entre 26 y 73 años de edad (Melton, Ellis, \& Zabriskie, 2016) y en otros grupos de contextos culturales diferentes, tales como estudiantes universitarios chilenos (Schnettler et al., 2017a) y adolescentes chilenos entre 10 y 17 años (Schnettler et al., 2017b). Respecto a las evidencias psicométricas de validez y confiabilidad, Zabriskie y Ward (2013), a partir de datos recolectados de adolescentes de diferentes culturas, señalan que la SWFLS, a partir de un análisis de componentes principales con rotación Varimax, presenta una estructura factorial unidimensional consistente, con cargas factoriales que varían de 0.7 a 0.93 y valores del coeficiente alfa de Cronbach que van desde 0.79 hasta 0.94 . Por tanto, brinda evidencia de que el instrumento es una opción breve, con propiedades psicométricas sólidas y ampliamente aplicable para la medición de la SWFLS. En el contexto latinoamericano, si bien el estudio de Schnettler et al. (2017b) menciona que se analizaron sus propiedades psicométricas para cada submuestra de padres e hijos adolescentes entre 10 y 17 años, los índices de ajuste al modelo reportados son aquellos derivados de un análisis factorial confirmatorio de los datos de la muestra total integrada por ambos padres y el hijo adolescente, y no solo de los adolescentes. Lo que si reportan los autores (Schnettler et al., 2017b) para la submuestra de adolescentes es un coeficiente de confiabilidad compuesto de 0.92 , $\mathrm{AVE}=0.71$ y cargas factoriales superiores a 0.58 y estadísticamente significativas.

A partir de la revisión de la literatura, ninguno de los análisis factoriales de la SWFLS previos ha comparado directamente las soluciones factoriales a partir del sexo, sin brindar evidencia para concluir que la escala mide el mismo concepto en hombres y mujeres y, por lo tanto, que puede interpretarse de la misma manera en ambos grupos. Establecer la invarianza factorial (IF) es un proceso importante para la demostración del adecuado funcionamiento de un instrumento de medición (Ventura-León, Barboza-Palomino, \& Caycho-Rodríguez, 2017). Así, verificar que las propiedades de medida de los instrumentos son independientes de las características de los grupos comparados, pero no del constructo que se está midiendo, es un requisito previo indispensable para la comparación de grupos con respecto a una variable latente (Byrne, 2008; Meredith, 1993; Widaman \& Reise, 1997). Un mayor grado de IF reportará que las diferencias observadas son resultado de las diferencias reales en el constructo evaluado (en este caso, satisfacción con la vida familiar) (Caycho-Rodríguez, 2017a), mientras que, si no se llega a demostrar IF, se hace probable la presencia de sesgo de medida a favor de alguno de los grupos comparados, lo cual afectaría la validez de las conclusiones del estudio (Byrne, 2008).

En este sentido, a pesar de que se ha usado en adolescentes latinoamericanos, una revisión de la literatura indica la existencia de pocos estudios que brinden evidencias psicométricas 
de la SWFLS en esta población. Ante esto, resulta pertinente plantear la siguiente pregunta de investigación: imostrará la SWFLS adecuadas propiedades psicométricas en adolescentes del Perú, en edad escolar? Por tanto, el objetivo del presente estudio es analizar las evidencias empíricas de validez de constructo, convergente, divergente, fiabilidad e invarianza factorial de la SWFLS, según el sexo, en una muestra peruana de adolescentes en edad escolar. Al considerar los argumentos anteriores, las hipótesis fueron las siguientes: (1) los cinco ítems de la SWFLS conformarán una estructura unidimensional; (2) se observarán correlaciones moderadas entre los cinco ítems de la SWFLS; (3) se presentarán correlaciones positivas entre la puntuaciones de la SWFLS y las de medidas de bienestar subjetivo, funcionalidad familiar y satisfacción académica; (4) la correlación entre las puntuaciones de la SWFLS y las medidas de depresión y ansiedad será negativa; (5) la magnitud del coeficiente de fiabilidad de las puntuaciones observadas y las variables latentes será moderada y por último, (6) las puntuaciones de los cinco ítems de la SWFLS serán invariantes según el sexo.

El presente estudio se justifica debido a la necesidad de contar con una medida breve de satisfacción con la vida familiar en la adolescencia, que sea de fácil aplicación e interpretación, que cuente además con evidencias de validez, confiabilidad y libre de sesgo entre hombres y mujeres; también, que pueda usarse en actividades orientadas al desarrollo de la calidad de vida familiar de los adolescentes. De igual manera, la SWFLS puede ser una herramienta útil para la medición empírica a nivel nacional y transnacional de la satisfacción con la vida familiar. Por tanto, la información derivada de la aplicación de la SWFLS permitirá una mejor comprensión de la satisfacción con la vida familiar desde la perspectiva de los adolescentes.

\section{Método}

\section{Participantes}

La muestra estuvo conformada por 804 estudiantes que cursaban del $6 .^{\circ}$ grado de primaria al $5 .^{\circ}$ grado de secundaria, en tres escuelas privadas ubicadas en la ciudad de Lima. Los participantes tenían edades comprendidas entre 11 y 18 años $(M=13.5, D E=1.6)$, donde 375 (46.6\%) eran mujeres y 429 (53.4 $\%)$ hombres. Los hombres tenían una edad promedio de 13.55 años $(D E=1.6)$, mientras que las mujeres 13.44 años $(D E=1.58)$. La comparación de medias evidenció la ausencia de diferencias significativas e importantes en el promedio de edad entre hombres y mujeres $(t$ (802) $=0.953, p=0.341, d=0.07$, IC95\% -0.07-0.2). De los participantes, 72 (9\%) cursaban el 6. grado de primaria; en el nivel secundario, 187 (23.3 \%) cursaban el primer grado, 153 (19\%) segundo grado, 157 (19.5\%) grado tercero, 126 (15.7\%) cuarto grado y 109 (13.6\%) quinto grado. El 63.9 \% de los participantes vivía con ambos padres, el $21.3 \%$ solo con la madre, el 4.6 $\%$ únicamente con el padre, el $9.8 \%$ con otros familiares y el $0.4 \%$ reportó vivir solo.

\section{Instrumentos}

Los participantes respondieron un cuestionario anónimo que comprendía datos sociodemográficos y los instrumentos que se enumeran a continuación.

Satisfaction with Family Life Scale ([SWFLS]; Zabriskie Eु McCormick, 2003)

Es una versión modificada de la SWLS (Diener et al., 1985), donde las palabras "vida familiar" reemplazaron el término "vida", en cada una de los cinco ítems originales. La SWFLS mide el juicio cognitivo consciente de la propia vida familiar con base en el grado de acuerdo o desacuerdo con las declaraciones globales sobre la vida familiar, en una escala tipo Likert de 
siete puntos $(1=$ totalmente en desacuerdo a 7 = totalmente de acuerdo). La SWFLS ha demostrado una buena consistencia interna de sus cinco ítems agrupados en una sola dimensión, en estudios previos de muestras de familias de Estados Unidos, Canadá, Reino Unido, Australia y Nueva Zelanda (Zabriskie \& Ward, 2013).

The World Health Organisation-Five WellBeing Index ([WHO-5 WBI]; World Health Organisation, 1998)

Cuestionario autoadministrado conformado por cinco ítems con cuatro alternativas de respuesta tipo Likert $(0=$ Nunca, $1=$ A veces, $2=$ Muchas veces, 3 = Siempre), que explora el bienestar general. La puntuación total se obtiene a partir de la sumatoria simple de las puntuaciones de cada ítem, donde puntajes altos expresan un mayor bienestar, mientras que puntajes menores serían indicador de la presencia de síntomas depresivos de importancia clínica. En el presente estudio, el valor del coeficiente alfa de Cronbach para estimar la confiabilidad fue de 0.73 (IC95\%, 0.7-0.76).

\section{Patient Health Questionnarie-2 ([PHQ-2]; Kroenke, Spitzer, $\mathcal{E}$ Williams, 2003)}

Consta de dos ítems con cuatro opciones de respuesta $(0=$ para nada a $3=$ hasta casi todos los días) que exploran aspectos emocionales y cognitivos asociados a la depresión (desánimo, desesperanza, displacer y desinterés en las cosas). El cálculo del coeficiente alfa de Cronbach como estimador de la confiabilidad para el presente estudio fue $\alpha=0.74$ (IC95\%, 0.71-0.77).

\section{APGAR Familiar (Smilkstein, 1978)}

Mide la percepción del funcionamiento familiar, a través de su satisfacción en las relaciones que tiene con sus familiares en cinco áreas específicas: adaptabilidad, cooperación, desarrollo, afectividad y capacidad resolutiva (adaptability, partnership, growth, affection, resolve). El cuestionario está conformado por cinco ítems con cinco opciones de respuesta tipo Likert $(0=$ nunca a $4=$ siempre $)$. Se usó la validación peruana realizada por Castilla, Caycho-Rodríguez, Ventura, BarbozaPalomino y De la Cruz (2015), donde las puntuaciones altas indican una buena percepción del funcionamiento familiar. La fiabilidad para este estudio, estimada mediante el valor del coeficiente alfa de Cronbach, es 0.8 (IC95\%, 0.77-0.82)

\section{Procedimiento}

El estudio fue aprobado por el Comité de Ética de la Universidad Privada del Norte. La versión original en inglés de la SWFLS fue traducida al español y luego nuevamente al inglés, considerando el método de traducción inversa recomendado internacionalmente (Acquadro, Jambon, Ellis, \& Marquis, 1996; Muñiz \& Hambleton, 1996). Así, dos profesionales de la salud mental bilingüe español/inglés tradujeron la versión original; discutidas ambas traducciones, se acordó una versión final en español que fue remitida a otros dos profesionales también especialistas para una traducción inversa al inglés. De igual manera, se discutieron estas traducciones y se determinó una última traducción inversa, que, mediante reuniones en busca de consenso respecto a su equivalencia lingüística, fue comparada con la original. La versión corregida fue aplicada a cinco adolescentes para las últimas correcciones y conseguir la versión final traducida.

Los estudiantes fueron invitados a participar voluntariamente, firmando ellos y sus padres o tutores el consentimiento informado. Entre junio y agosto de 2017, los participantes, luego de recibir las instrucciones, completaron los cuestionarios en sus aulas de clases y en presencia de un conjunto de estudiantes de psicología especialmente entrenados. La mayoría de los participantes respondió los cuestionarios de forma independiente. 


\section{Resultados}

\section{Análisis de datos}

Se calcularon estadísticos descriptivos (media, desviación estándar, asimetría y curtosis) para los cinco ítems de la SWFLS y el coeficiente Mardia (1970) de normalidad multivariada. Por otra parte, el análisis factorial confirmatorio (AFC) se realizó utilizando el programa $\mathrm{R}$ ( $\mathrm{R}$ Development Core Team, 2007), con el objetivo de examinar el ajuste de un modelo unidimensional donde todos los ítems carguen en una sola variable latente, y con base en la matriz de correlaciones policóricas y la estimación de máxima verosimilitud (Hair, Anderson, Tatham, \& Black, 2014). La bondad del ajuste del modelo se efectuó con la prueba chi-cuadrad $\left(\chi^{2}\right)$, considerando que esta es sensible al tamaño muestral (Fujikoshi, 2000). Se reportó la chi-cuadrada relativa $\chi^{2} / d f$ (Bollen, 1998), que expresa un ajuste de modelo adecuado al presentar valores entre 2 y 3 , o de manera más flexible, si presenta valores $\leq 5$ (Carmines \& Mclver, 1981; Jöreskog, 1970). Se estimaron: el índice de bondad de ajuste (GFI), el índice de ajuste comparativo (CFI), el índice de ajuste normativo (NFI), el error cuadrático medio de aproximación (RMSEA) y el residuo cuadrático medio estandarizado (SRMR). Se utilizaron los valores indicativos de buen ajuste, los cuales son para el GFI, CFI y NFI > 0.9, RMSEA y SRMR < 0.08 (Hu \& Bentler, 1999). En relación con las cargas factoriales estandarizadas $(\lambda)$ se consideraron valores $\geq 0.5$ como adecuados (Johnson \& Stevens, 2001). Se calculó también la varianza promedio extraída del factor Average Variance Extracted (AVE), cuyo valor superior a 0.5 brinda evidencia de validez interna convergente (Bhattacherjee \& Premkumar, 2004; Fornell \& Larcker, 1981; Wixom \& Todd, 2005).

Para determinar si la SWFLS es invariante en relación con el sexo, se evaluó progresivamente la invarianza configural (M1: invarianza de la estructura de la SWFLS entre los grupos), invarianza métrica (M2: invariancia de las cargas factoriales entre los grupos), invarianza fuerte
(M3: invarianza de los interceptos entre los grupos) e invarianza estricta (M4: la invarianza de los residuos se añade a la invarianza de cargas factoriales e interceptos) mediante un AFC multigrupo (Byrne, 2008; Chen, 2007). En este tipo de análisis, se establece una serie de restricciones cada vez más estrictas sobre los parámetros del modelo para evaluar su igualdad entre hombres y mujeres. Para la evaluación de las diferencias entre los modelos, se emplearon las variaciones de los índices CFI $(\Delta \mathrm{CFI})$ y RMSEA ( $\triangle$ RMSEA). La invarianza fuerte se admite cuando $\Delta$ CFI $\leq 0.01$ y $\triangle$ RMSEA $\leq 0.015$ (Cheung \& Rensvold, 2002).

Las evidencias de validez convergente y discriminante se obtuvieron al calcular los coeficientes de correlación de Pearson ( $r$ ) entre la puntuación de la SWFLS y otras medidas de depresión (PHQ-2), bienestar general (WHO-5 WBI) y funcionalidad familiar (APGAR) mediante el SPSS 25 (2017). Se calcularon los IC de los valores $r$ utilizando un módulo MS Excel® ad hoc y se interpretó el tamaño del efecto (TE) de las correlaciones en función del valor de $r$, donde $r \geq 0.2, r \geq 0.5 \mathrm{y} r \geq$ 0.8 expresan un TE mínimo, moderado y fuerte, respectivamente (Caycho-Rodríguez, 2017b).

Para la estimación de la confiabilidad de las puntuaciones observadas se calculó el coeficiente alfa de Cronbach ( $\alpha$ ) (1951) con sus intervalos de confianza (IC) (Domínguez-Lara \& MerinoSoto, 2015), mientras que para las variables latentes se calculó el valor del coeficiente Omega (w) (McDonald, 1999; Ventura-León \& CaychoRodríguez, 2017) con sus IC (Ventura-León, 2017) y el coeficiente $H$ (Domínguez-Lara, 2016; Hancock \& Mueller, 2001). Se esperan magnitudes $>0.8$ para el coeficiente $\alpha$ (Merino, Navarro, \& García, 2014), w y H (Raykov \& Hancock, 2005).

\section{Análisis descriptivo de los ítems de la SWFLS}

La Tabla 1 muestra que el ítem 3 tiene el mayor puntaje promedio $(\mathrm{M}=5.4)$ y el ítem 5 el más bajo $(M=4.67)$. En relación con la variabilidad, el ítem $5(\mathrm{DE}=2.05)$ muestra 
la mayor dispersión. Los valores de asimetría y curtosis de los cinco ítems de la SWFLS se encuentran por debajo del límite esperado $(<$ \pm 1.5). El valor del coeficiente de Mardia, con un valor de 8.09, brinda evidencia para el cumplimiento del supuesto de normalidad multivariada (Rodríguez \& Ruiz, 2008), ya que es inferior a 35 de acuerdo a lo sugerido por Bollen (1989) con base en la fórmula $\mathrm{p}(\mathrm{p}+2)$, donde $\mathrm{p}$ es la cantidad de variables observadas. Los hallazgos no afectarían las estimaciones posteriores (Ferrando \& Anguiano-Carrasco, 2010; Pérez, \& Medrano, 2010).

\section{Tabla 1}

Medias, desviaciones estándar, asimetría, curtosis y correlación entre ítems de la SWFLS

\begin{tabular}{|c|c|c|c|c|c|c|c|c|}
\hline \multirow{2}{*}{ İtem } & \multirow{2}{*}{$\begin{array}{c}M \\
\text { (IC95\%) }\end{array}$} & \multirow{2}{*}{$D E$} & \multirow{2}{*}{$\mathrm{g} 1$} & \multirow{2}{*}{ g2 } & \multicolumn{4}{|c|}{ Correlación inter-ítem } \\
\hline & & & & & Ítem 1 & Ítem 2 & Ítem 3 & Ítem 4 \\
\hline Ítem 1 & $\begin{array}{c}4.79 \\
(4.65-4.94)\end{array}$ & 1.6 & -0.57 & -0.25 & & & & \\
\hline Ítem 2 & $\begin{array}{c}4.99 \\
(4.84-5.13)\end{array}$ & 1.58 & -0.71 & -0.2 & 0.52 & & & \\
\hline Ítem 3 & $\begin{array}{c}5.4 \\
(5.26-5.55)\end{array}$ & 1.62 & -0.93 & 0.06 & 0.54 & 0.62 & & \\
\hline Ítem 4 & $\begin{array}{c}4.84 \\
(4.69-4.99)\end{array}$ & 1.63 & -0.66 & -0.25 & 0.47 & 0.54 & 0.57 & \\
\hline Ítem 5 & $\begin{array}{c}4.67 \\
(4.48-4.85)\end{array}$ & 2.05 & -0.39 & -1.17 & 0.43 & 0.49 & 0.52 & 0.45 \\
\hline
\end{tabular}

Nota. $\mathrm{M}=$ Media; $\mathrm{DE}=$ Desviación Estándar; $\mathrm{g} 1$ = Asimetría; $\mathrm{g} 2$ = Curtosis. Ítem 1 = "En

la mayoría de los aspectos, mi vida familiar está próxima a mi ideal"; Ítem 2 = "Las condiciones de mi vida familiar son excelentes"; Ítem 3 = "Estoy satisfecho(a) con mi vida familiar"; Ítem 4 = "Hasta ahora, he logrado las cosas importantes que quería en mi vida familiar"; Ítem $5=$ "Si pudiera vivir mi vida familiar otra vez, no cambiaría casi nada".

\section{Análisis de la estructura interna de la SWFLS}

Para el AFC se cargaron los cinco ítems de la SWFLS, en una sola variable latente (Tabla 2). Los índices de bondad de ajuste revelaron que el modelo de factor único encajaba bien con los datos $\left(\chi^{2}=3.49, d f=5, p=0.63 ; \chi^{2} / d f=0.69\right.$; $\mathrm{GFI}=0.998$; CFI $=1$; NFI $=0.998$; RMSEA $=0[\operatorname{IC90\% } 0,0.038]$ y SRMR $=0.007$ ). Las cargas factoriales estandarizadas $(\lambda)$ para el modelo unidimensional fueron significativas y en la dirección esperada (ítem $1=0.672$; ítem $2=0.77$; ítem $3=0.807$; ítem $4=$ 0.701 ; ítem $5=0.642)$ con una $\lambda$ promedio de 0.718, superior al 0.7 requerido (Hair et al., 2014). Asimismo, las correlaciones entre los ítems de la SWFLS (Tabla 1) no son mayores a 0.9 (no existe multicolinealidad) y el valor de $\mathrm{AVE}=0.52(>0.5)$, permitiendo tener evidencia empírica de validez interna discriminante (Tabachnick, Fidell, \& Osterlind, 2001) y validez interna convergente (Fornell \& Larcker, 1981), respectivamente.

\section{Tabla 2}

Estructura interna e invarianza factorial de la SWFLS

\begin{tabular}{|c|c|c|c|c|c|c|c|c|}
\hline Modelo & $\begin{array}{c}\chi^{2} \\
(g l)\end{array}$ & $\begin{array}{l}\Delta \chi^{2} \\
(\Delta g l)\end{array}$ & $\begin{array}{l}\text { RMSEA } \\
{[\text { [IC } 90 \%]}\end{array}$ & $p$ & SRMR & CFI & $(\Delta \mathrm{CFI})$ & (ARMSEA) \\
\hline Todo el grupo & $\begin{array}{l}3.492 \\
(5)^{*}\end{array}$ & - & $\begin{array}{c}0 \\
{[0,0.038]}\end{array}$ & 0.625 & 0.007 & 1 & - & \\
\hline Mujeres & $\begin{array}{c}6.997 \\
(5)\end{array}$ & - & $\begin{array}{c}0.033 \\
{[0,0.084]}\end{array}$ & 0.221 & 0.015 & 0.997 & - & \\
\hline Hombres & $\begin{array}{l}4.183 \\
(5)\end{array}$ & - & $\begin{array}{c}0 \\
{[0,0.061]}\end{array}$ & 0.523 & 0.011 & 1 & - & \\
\hline M1 & $\begin{array}{c}11.18 \\
(10)\end{array}$ & - & $\begin{array}{c}0.017 \\
{[0,0.058]}\end{array}$ & - & 0.013 & 0.999 & - & - \\
\hline M2 & $\begin{array}{c}16.676 \\
(14)\end{array}$ & $\begin{array}{c}5.495 \\
(4)\end{array}$ & $\begin{array}{c}0.022 \\
{[0,0.055]}\end{array}$ & 0.274 & 0.028 & 0.998 & 0.001 & 0.005 \\
\hline M3 & $\begin{array}{c}11.18 \\
(10)\end{array}$ & $\begin{array}{c}2.002 \\
\text { (4) }\end{array}$ & $\begin{array}{c}0.01 \\
{[0,0.046]}\end{array}$ & 0.412 & 0.03 & 1 & 0.001 & 0.012 \\
\hline M4 & $\begin{array}{c}28.338 \\
(23)\end{array}$ & $\begin{array}{l}9.658 \\
(5)\end{array}$ & $\begin{array}{c}0.024 \\
{[0,0.05]}\end{array}$ & 0.203 & 0.033 & 0.996 & 0.003 & 0.014 \\
\hline
\end{tabular}

Análisis de la invarianza factorial de la SWFLS según sexo

Los índices de ajuste para el modelo unidimensional (para cada sexo por separado y en la muestra total) se muestran en la Tabla 2. Con base en los índices de ajuste global, se obtiene apoyo estadístico para el modelo unidimensional en ambos grupos. Para la determinación de la IF según sexo se evaluó progresivamente la invarianza configuracional (M1), la invarianza métrica (M2), la invarianza fuerte (M3) y la invarianza estricta (M4) a partir del modelo unidimensional establecido (Byrne, 2008).

En primer lugar, se analizó la estructura de la SWFLS entre ambos sexos (M1), presentando como resultado excelentes valores de $\mathrm{CFI}=$ 0.999, SRMR $=0.013$ y RMSEA $=0.017$ $(\mathrm{IC} 90 \%=0,0.058)$, además de cargas factoriales significativas $(p<0.001)$. Los resultados demuestran que el modelo unidimensional se ajusta adecuadamente a los datos en ambos grupos. En segundo lugar, tomando como referencia M1 se probó el M2 donde las cargas 
factoriales son iguales en todos los grupos. Los resultados mostraron que $\mathrm{M} 2$ se ajusta bien con índices RMSEA $=0.022(\mathrm{IC} 90 \%=0,0.055)$, $\mathrm{SRMR}=0.028$ y CFI $=0.998$. Al comparar M2 y $\mathrm{M} 1$, no se observan cambios significativos $(\triangle \mathrm{CFI}$ $=0.001$ y $\Delta$ RMSEA $=0.005)$. Los hallazgos sugieren que las cargas factoriales son invariantes entre los grupos de hombres y mujeres.

En tercer lugar, se evaluó M3, donde las cargas factoriales e interceptos son iguales entre los grupos de mujeres y hombres. Los resultados indican que M3 muestra adecuados índices de ajuste (RMSEA $=0.01[$ IC90\% $=0,0.046]$, $\mathrm{SRMR}=0.03, \mathrm{CFI}=1) . \mathrm{Al}$ comparar M2 y M3 no se evidencian cambios significativos $(\Delta$ CFI $=0.001$ y $\Delta$ RMSEA $=0.012)$, lo que permite aceptar la hipótesis de que los interceptos son invariantes entre ambos grupos. Finalmente, se analizó M4 donde las cargas factoriales, interceptos y residuos se mantienen invariantes en ambos grupos, reportando adecuados índices de ajuste (RMSEA $=0.024$ [IC90\% $=0,0.05$ ], $\mathrm{SRMR}=0.033, \mathrm{CFI}=0.996)$. En comparación con los índices de ajuste de M3, las diferencias son las esperadas $(\triangle \mathrm{CFI}=0.003$ y $\triangle$ RMSEA $=0.014)$, brindando apoyo empírico para la invarianza estricta. En conjunto, los resultados sugieren que la invarianza factorial de la SWFLS se mantiene según sexo.

\section{Confiabilidad}

La confiabilidad para variables latentes fue $\omega$ $=0.84(\mathrm{IC} 95 \%=0.81-0.85)$ y $\mathrm{H}=0.85$. En relación a las puntuaciones observadas se reporta un $\alpha=0.84$ (IC95\% $=0.82-0.86)$. Los resultados indican una confiabilidad adecuada del modelo unidimensional de la SWFLS.

Validez convergente y discriminante

Para examinar la validez convergente $y$ discriminante de la SWFLS, se calcularon los coeficientes de correlación de Pearson entre SWFLS, PHQ-2, WHO-5 WBI y APGAR. Como se esperaba, la SWFLS se correlacionó negativamente de manera significativa con
PHQ-2 $(r=-0.369, p<0.01[$ IC95\% $=0.31$, 0.59]). Así también, se reportaron correlaciones positivas y significativas entre la SWFLS y WHO-5 WBI $(r=0.463, p<0.01$ [IC95\% $=0.41,0.68])$ y APGAR $(r=0.655, p$ $<0.01$ [IC95\% $=0.61,0.82]$ ). Todas las correlaciones presentan un TE pequeño $(r \geq 0.2)$ y moderado $(r \geq 0.5)$ (Caycho-Rodríguez, 2017). Los resultados presentan evidencias de validez convergente y divergente aceptable.

\section{Discusión}

El propósito del presente estudio fue examinar las evidencias de validez, confiabilidad y la invarianza de medición a través del sexo de la versión peruana de la SWFLS para contar con una medida adecuada en escolares peruanos, que puede ser utilizada en contextos de investigación. Al considerar los resultados, la SWFLS presenta un funcionamiento óptimo.

En relación con el análisis de la estructura interna, los resultados apoyan la estructura unidimensional de la SWFLS, que es consistente con lo señalado por Zabriskie y Ward (2013) en muestras de Estados Unidos, Canadá, Reino Unido, Australia y Nueva Zelanda y Schnettler et al. (2017b), con adolescentes chilenos entre $10 \mathrm{y}$ 17 años. Este resultado brinda sustento empírico para interpretar el constructo satisfacción con la vida familiar desde un punto de vista unitario, cumpliendo así un requerimiento importante para un instrumento de medida, que es el de medir un único rasgo latente (Wilson, 2005). Además, si bien las cargas factoriales de los ítems de la versión peruana de la SWFLS son superiores a lo requerido ( $\geq 0.5$; Johnson \& Stevens, 2001) y similares a lo señalado en adolescentes chilenos (Schnettler et al., 2017b), dos de los cinco ítems (ítem 1 e ítem 5) son menores a lo reportado a nivel internacional (de 0.7 a 0.93 ; Zabriskie \& Ward, 2013). Las diferencias en las cargas factoriales, evidencian que ambos ítems (1 y 5) influyen de manera diferente en la definición del constructo satisfacción con la vida familiar. Con esto se considera que, para la muestra de escolares peruanos, una adecuada representación 
de la puntuación de la SWFLS estaría asociada con una ponderación desigual de cada ítem (Merino-Soto \& Salas-Blas, 2017).

Respecto a la confiabilidad, tanto el análisis para las variables latentes $(\omega=0.84$ y $\mathrm{H}=0.85)$ y las puntuaciones observadas $(\alpha=0.84)$, indica que los ítems son homogéneos (Muñiz, 1999), lo cual está dentro de los límites reportados por Zabriskie y Ward (2013) en muestras de diversos países de América, Europa y Oceanía ( $\alpha$ de 0.79 hasta 0.94). Por otro lado, los valores de los coeficientes de confiabilidad del presente estudio son menores a lo registrado por el coeficiente de confiabilidad compuesto de 0.92 , reportado en un estudio con adolescentes chilenos (Schnettler et al., 2017b). Los resultados son los esperados para medidas breves de tamizaje (DomínguezLara \& Merino-Soto, 2017) y suficientes para su uso en contextos de investigación, pero no para la toma de decisiones en el ámbito clínico o aplicado en general, mediante las cuales se afecte la vida de las personas, lo cual exige valores de coeficiente de confiabilidad $>0.9$ (DeVellis, 2003; Nunnally \& Bernstein, 1995; Rosenthal, 1994). Así, para mediciones más precisas de la satisfacción con la vida familiar es necesario medidas con mayor cantidad de ítems o una combinación de instrumentos.

Es importante destacar que el presente estudio es el primero que examina la invariancia por sexo de la SWFLS. De esta manera, la invariancia configuracional, métrica, fuerte y estricta de la SWFLS se mantuvo al comparar los grupos de hombres y mujeres. Esto sugiere que las variables observadas (ítems) miden la misma variable latente en formas idénticas entre ambos sexos (Brown, 2006). Así, desde un punto de vista psicométrico, la IF es un requisito previo a la realización de cualquier comparación entre grupos por sexo (Byrne, 2008; Meredith, 1993). Cuando los datos apoyan la estructura unidimensional subyacente de la SWFLS como invariante por sexo, es posible afirmar que los adolescentes peruanos en edad escolar (hombres y mujeres) conceptualizan la satisfacción con la vida familiar de la misma manera, lo que les permite interpretar y responder a los ítems de la SWFLS de forma similar, que la estructura factorial reportada es semejante y que es presentada en la misma métrica en todos los grupos. Además, si existe alguna diferencia en la puntuación media latente de la SWFLS, puede explicarse con la seguridad de que esa diferencia es resultado de la verdadera diferencia en la variable latente y no por un sesgo de la medida en sí.

Para dar evidencias de validez convergente y discriminante, se analizó la asociación entre las medidas de SWFL: depresión, ansiedad, funcionalidad familiar, bienestar y satisfacción con los estudios. La satisfacción con la vida familiar se asocia positivamente con el bienestar subjetivo y la funcionalidad familiar y negativamente con la depresión, siendo similar a los hallazgos en estudios previos (Luna, 2012; Luna, Laca, \& Mejía, 2011; Stavropoulos, Lazaratou, Marini, \& Dikeos, 2015). Estos resultados sugieren que la SWFL asociada con la percepción del buen funcionamiento familiar (adecuada cohesión familiar, apoyo de los miembros de la familia, ausencia de conflictos, etc.) es una variable importante para el logro de una vida feliz y plena sin la aparición de un conjunto de síntomas depresivos, en los adolescentes (Ferro \& Boyle, 2015; Gladstone, Parker, Mitchell, Wilhelm, \& Malhi, 2005; Sheeber, Davis, Leve, Hops, \& Tildesley, 2007; Shittu et al., 2014). Los hallazgos son importantes desde un punto de vista práctico, pues una disminución de la satisfacción familiar puede ser explicada por la percepción de disfuncionalidad familiar y la presencia de síntomas depresivos, lo cual resulta ser más frecuente en la adolescencia (Safer, 2009). Es posible que respuestas familiares inadecuadas a las necesidades de los adolescentes induzcan posteriormente a sensaciones de baja satisfacción familiar (Painuly, Sharan, \& Mattoo, 2005). Es importante mencionar que existe la posibilidad de que estas relaciones tengan diferente intensidad en aquellas culturas donde la familia se valora significativamente, como es el caso contexto cultural peruano.

El estudio presenta algunas limitaciones. En primer lugar, debido a razones de accesibilidad, solo se incluyeron los escolares de instituciones 
educativas privadas, mientras que aquellos de las públicas no fueron considerados. De esta manera, la generalización de los resultados ha podido verse afectada debido a las características de los participantes. Segundo, la medición de la satisfacción con la vida familiar se realizó transversalmente, por lo que estudios longitudinales brindarían mayor evidencia de validez predictiva. En tercer lugar, la confiabilidad test-retest no se examinó en esta investigación.

En conclusión, los resultados del análisis de confiabilidad y AFC de la versión peruana de la SWFLS muestran que esta tiene excelentes propiedades psicométricas para medir la satisfacción con la vida familiar en escolares peruanos de educación secundaria. Sin embargo, es necesario realizar más estudios psicométricos en diversas poblaciones de escolares. En este sentido, investigaciones futuras deberían avanzar en el estudio de la IF de la SWFLS según grupos de edad, nivel socioeconómico, grupo étnico, etc., con el fin de garantizar la comparabilidad y la equivalencia del constructo satisfacción con la vida familiar. De igual forma, el empleo de medidas de autoinformes como la SWFLS en estudios a escala nacional, dada su fácil y rápida administración, representa una línea de investigación altamente prometedora.

\section{Agradecimientos}

Los autores agradecen a Jennifer D. CasariegoOviedo, Piero Nuñez-Apolinario, Marilyn Cuellar-Llata, Valeria Franco-Reynoso, Melanie Mendives-Delgado, Jahaira Carranza-Fuentes, Fiorella A. Torrejón-Pinedo, Lisseth Rojas-Ayala por su colaboración en la recolección de la información para este estudio.

\section{Referencias}

Acquadro, C., Jambon, B., Ellis, D., \& Marquis, P. (1996). Language and translation issues. En B. Spilker (Ed.), Quality of life and pharmacoeconomics in clinical trials (pp.
575-585). Philadelphia, PA: LippincottRaven.

Andrews, F. M., \& Withey, S. B. (1976). Social indicators of well-being: Americans' perceptions of life quality. Nueva York: Plenum.

Antaramian, S. P., Huebner, E. S., Hills, K. J., \& Valois, R. F. (2010). A dualfactor model of mental health: Toward a more comprehensive understanding of youth functioning. American Journal of Orthopsychiatry, 80(4), 462-472. https://doi .org/10.1111/j.1939-0025.2010.01049.x

Arias, W. L., Quispe, A. C., \& Ceballos, K. D. (2016). Estructura familiar y nivel de logro de niños y niñas de escuelas públicas de Arequipa. Perspectiva de Familia, 1, 35-62. Recuperado de http://ucsp.edu.pe/imf/wp-content/uplo ads/2016/10/3.-Estructura-familiar-y-logrode-aprendizaje.pdf

Arnett, J. J. (2008). Adolescencia y adultez emergente. Un enfoque cultural (3.a ed.). México: Pearson Educación.

Balaguer, I., Castillo, I., \& Duda, J. L. (2008). Apoyo a la autonomía, satisfacción de las necesidades, motivación y bienestar en deportistas de competición. Un análisis de la teoría de autodeterminación. Revista de Psicología del Deporte, 1(17), 123-139. Recuperado de http://www.rpd-online.com /article/view/246/246

Barboza-Palomino, M., Moori, I., Zárate, S., López, A., Muñoz, K., \& Ramos, S. (2017). Influencia de la dinámica familiar percibida en el proyecto de vida en escolares de una institución educativa de Lima [Influência da dinâmica familiar percebida no projeto de vida de alunos de uma escola de Lima]. Psicologia Escolar e Educacional, 21(2), 157-166. https://doi.org/10.1590/2175-353 9201702121094

Barraca, J., López Yarto, L., \& Olea, J. (2000). Psychometric properties of a new Family Life Satisfaction Scale. European Journal of Psychological Assessment, 16(2), 98-106. htt ps://doi.org/10.1027/1015-5759.16.2.98 
Bazo-Alvarez, J. C., Bazo-Alvarez, O. A., Aguila, J., Peralta, F., Mormontoy, W., \& Bennett, I. M. (2016). Propiedades psicométricas de la Escala de Funcionalidad Familiar FacesIII: un estudio en adolescentes peruanos. Revista Peruana de Medicina Experimental y Salud Publica, 33(3), 462-470. https://doi.o rg/10.17843/rpmesp.2016.333.2299

Bhattacherjee, A., \& Premkumar, G. (2004). Understanding changes in belief and attitude toward information technology usage: A theoretical model and longitudinal test. MIS Quarterly, 28(2), 229-254. https:/ /doi.org/10.2307/25148634

Bollen, K. A. (1989). Structural equations with latent variables. Nueva York: Wiley.

Bollen, K. A. (1998). Structural equation models. En P. Armitage \& T. Colton (Eds.), Encyclopedia of Biostatistics (pp. 43634372). Sussex, UK: Wiley.

Brown, T. A. (2006). Confirmatory factor analysis for applied research. Nueva York: Guilford Press.

Byrne, B. M. (2008). Testing for multigroup equivalence of a measuring instrument: A walk through the process. Psicothema, 20(4), 872-882. Recuperado de http://www.psicothema.com/english/psi cothema.asp? $\mathrm{id}=3569$

Çakar, F. F., Tagay, Ö. O., \& Karataş, Z. Z. (2015). Adolescents' life satisfaction: Risky behaviors and hopelessness. International Journal on New Trends in Education $\mathcal{B}$ Their Implications, 6(1), 55-62. Recuperado de http://www.ijonte.org/FileUpload/ks632 07/File/ijonte_2015.1.complete.pdf

Campbell, A., Converse, P. E., \& Rodgers, W. L. (1976). The quality of American life: Perceptions, evaluations, and satisfactions. Nueva York: Russell Sage Foundation.

Carver, M. D., \& Jones, W. H. (1992). The Family Satisfaction Scale. Social Behavior and Personality: An International Journal, 20(2), 71-83. https://doi.org/10.2224/sbp.1 992.20.2.71

Castilla, H., Caycho-Rodríguez, T., Ventura, J. L., Barboza-Palomino, M., \& De la Cruz, M. (2015). Análisis factorial confirmatorio de la Escala de Percepción del Funcionamiento Familiar de Smilkstein en adolescentes peruanos. Salud Eु Sociedad, 6(2), 140-153. https://doi.org/10.22199/SO 7187475.2015.0002.00003

Carmines, E. G., \& McIver, J.P. (1981). Analyzing models with unobservable variables. En G. Bohrnstedt \& E. Borgatta (Eds.), Social measurement: Current issues (pp. 65-115). Beverly Hills, CA: Sage.

Caycho-Rodríguez, T. (2017a). Importancia del análisis de invarianza factorial en estudios comparativos en Ciencias de la Salud. Educación Médica Superior, 31(2), 1-3. Recuperado de http://ems.sld.cu/index.php /ems/article/view/1043

Caycho-Rodríguez, T. (2017b). Tamaño del efecto e intervalos de confianza para correlaciones: aportes a Montes Hidalgo y Tomás-Sábado. Enfermería Clínica, 27(5), 331-332. https://doi.org/10.1016/j.enfcli.20 17.07 .001

Chen, F. F. (2007). Sensitivity of goodness of fit indices to lack of measurement invariance. Structural Equation Modeling: A Multidisciplinary Journal, 14(3), 464-504. https://doi.org/10.1207/s1532800 7sem1203_7

Cheung, G. W., \& Rensvold, R. B. (2002). Evaluating goodness-of-fit indexes for testing measurement invariance. Structural Equation Modeling: A Multidisciplinary Journal, 9(2), 233-255. https://doi.org/10.1 207/S15328007SEM0902_5

Cronbach, L. J. (1951). Coefficient alpha and the internal structure of tests. Psychometrika, 16(3), 297-334. https://doi.org/10.1007/BF 02310555

DeVellis, R. F. (2003). Scale Development. Theory and applications (2.a ed.). Thousand Oaks, CA: Sage.

Diener, E., Emmons, R. A., Larsen, R. J., \& Griffin, S. (1985). The Satisfaction with Life Scale. Journal of Personality Assessment, 49(1), 71-75. https://doi.org/10.1207/s1532 7752jpa4901_13

Diener, E., \& Ryan, K. (2009). Subjective wellbeing: A general overview. South African 
Journal of Psychology, 39(4), 391-406. https: //doi.org/10.1177/008124630903900402

Domínguez-Lara, S. A. (2016). Evaluación de la confiabilidad del constructo mediante el coeficiente $\mathrm{H}$ : breve revisión conceptual y aplicaciones. Psychologia. Avances de la Disciplina, 10(2), 87-94. https://doi.org/10. 21500/19002386.2134

Domínguez-Lara, S. A., \& Merino-Soto, C. (2015). ¿Por qué es importante reportar los intervalos de confianza del coeficiente alfa de Cronbach? Revista Latinoamericana de Ciencias Sociales, Niñez y Juventud, 3(2), 1326-1328. Recuperado de http://revistau manizales.cinde.org.co/rlcsnj/index.php/Re vista-Latinoamericana/article/view/2030

Domínguez-Lara, S. A., \& Merino-Soto, C. (2017). Versión breve de la Escala de Afrontamiento ante la Ansiedad e Incertidumbre Preexamen (COPEAU) en universitarios peruanos. Educación Médica. https://doi.org/10.1016/ j.edumed.2017.04.011

Easterlin, R. A. (2006). Life cycle happiness and its sources: Intersections of psychology, economics, and demography. Journal of Economic Psychology, 27(4), 463-482. https: //doi.org/10.1016/j.joep.2006.05.002

Emerson, S. D., Guhn, M., \& Gadermann, A. M. (2017). Measurement invariance of the Satisfaction with Life Scale: Reviewing three decades of research. Quality of Life Research, 26(9), 2251-2264. https://doi.org/ 10.1007/s11136-017-1552-2

Ferrando, P. J., \& Anguiano-Carrasco, C. (2010). El análisis factorial como técnica de investigación en psicología. Papeles del Psicólogo, 31(1), 18-33. Recuperado de http://www.papelesdelpsicologo.es/pdf/1 793.pdf

Ferro, M. A., \& Boyle, M. H. (2015). The impact of chronic physical illness, maternal depressive symptoms, family functioning, and self-esteem on symptoms of anxiety and depression in children. Journal of Abnormal Child Psychology, 43(1), 177-187. https://do i.org/10.1007/s10802-014-9893-6
Fornell, C., \& Larcker, D. F. (1981). Evaluating structural equation models with unobservable variables and measurement error. Journal of Marketing Research, 18(1), 39-50. https://doi.org/10.2307/3151312

Frasquilho, D., Matos, M., Neville, F., Gaspar, T., \& Almeida, J. (2016). Parental unemployment and youth life satisfaction: The moderating roles of Satisfaction with Family Life. Journal of Child $\mathscr{E}$ Family Studies, 25(11), 3214-3219. https://doi.org/ $10.1007 / \mathrm{s} 10826-016-0480-z$

Fujikoshi, Y. (2000). Transformations with improved chi-squared approximations. Journal of Multivariate Analysis, 72(2), 249-263. https://doi.org/10.1006/jmva.199 9.1854

Gilman, R., \& Huebner, E. S. (2003). A review of life satisfaction research with children and adolescents. School Psychology Quarterly, 18(2), 192-205. https://doi.org/10.1521/scp q.18.2.192.21858

Gilman, R., \& Huebner, E. S. (2006). Characteristics of adolescents who report very high life satisfaction. Journal of Youth and Adolescence, 35(3), 293-301. https://do i.org/10.1007/s10964-006-9036-7

Gladstone, G. L., Parker, G. B., Mitchell, P. B., Wilhelm, K. A., \& Malhi, G. S. (2005). Relationship between selfreported childhood behavioral inhibition and lifetime anxiety disorders in a clinical sample. Depression and Anxiety, 22(3), 103-113. https://doi.org/10.1002/da.20082

González, N., Valdez, J., \& Zavala, Y. (2008). Resiliencia en adolescentes mexicanos. Enseñanza e Investigación en Psicología, 13(1), 41-52. Recuperado de https:/www.researchgate.net/publicatio n/40441109_Resiliencia_en_adolescentes_ mexicanos

González-Serrano, G., Huéscar Hernández, E., \& Moreno-Murcia, J. A. (2013). Satisfacción con la vida y ejercicio físico. Motricidad. European Journal of Human Movement, 30, 131-151. Recuperado de https://dialnet.uni rioja.es/servlet/articulo? codigo $=4775597$ 
Guerney, B. G., Jr. (1977). Relationship enhancement: Skill training programs for therapy, problem prevention, and enrichment. San Francisco, CA: Jossey-Bass.

Guillén, R., Aliaga, K., Quispe, F., Nicolás, Y., Solís, R., Robles, Y., ... Vilchez, L. (2011). Adaptación de la Escala de Satisfacción Familiar por Adjetivos (ESFA) en universitarios de Lima metropolitana. Anales de Salud Mental, 27(2), 14-21. Recuperado de http://www.insm.gob.pe/ojsinsm/index.p $\mathrm{hp} /$ Revista1/article/view/83/64

Hair, J., Anderson, R., Tatham, R., \& Black, W. (2014). Análisis multivariante. México: Prentice Hall.

Hancock, G. R., \& Mueller, R. O. (2001). Rethinking construct reliability within latent variable systems. En R. Cudeck, S. H. C. du Toit \& D. Sörbom (Eds.), Structural equation modeling: Past and present. A Festschrift in honor of Karl G. Jöreskog (pp. 195-261). Chicago: Scientific Software International.

Hu, L. T., \& Bentler, P. M. (1999). Cutoff criteria for fit indexes in covariance structure analysis: Conventional criteria versus new alternatives. Structural Equation Modeling: A Multidisciplinary Journal, 6(1), 1-55. http s://doi.org/10.1080/10705519909540118

Huebner, E. S. (2004). Research on assessment of life satisfaction of children and adolescents. Social Indicators Research, 66, 3-33. https:// doi.org/10.1007/978-1-4020-2312-5_2

Huebner, E. S., Suldo, S. M., \& Gilman, R. (2006). Life Satisfaction. En G. G. Bear \& K. M. Minke (Eds.), Children's Needs III: Development, prevention, and intervention (pp. 357-368). Washington, DC: National Association of School Psychologists.

Jianfeng, T., Wu, Y., Hongwei, M., \& Yulan, Y. (2016). Adolescents' core self-evaluations as mediators of the effect of mindfulness on life satisfaction. Social Behavior $\mathbb{E}$ Personality: An International Journal, 44(7), 1115-1122. https://doi.org/10.2224/sbp.20 16.44.7.1115
Johnson, B., \& Stevens, J. J. (2001). Exploratory and confirmatory factor analysis of the School Level Environment Questionnaire (SLEQ). Learning Environments Research, 4(3), 325-344. https://doi.org/10.1023/A:1 014486821714

Jöreskog, K. G. (1970). A general method for analysis of covariance structures. Biometrika, 57(2), 239-251. https://doi.org/ 10.1093/biomet/57.2.239

Kroenke, K., Spitzer, R. L., \& Williams, J. B. (2003). The Patient Health Questionnaire-2: Validity of a two-item depression screener. Medical Care, 41(11), 1284-1292. https://doi.org/10.1097/01.ML R.0000093487.78664.3C

Lewis, A. D., Huebner, E. S., Malone, P. S., \& Valois, R. F. (2011). Life satisfaction and student engagement in adolescents. Journal of Youth and Adolescence, 40(3), 249-262. h ttps://doi.org/10.1007/s10964-010-9517-6

Luna, A. C. A. (2012). Funcionamiento familiar, conflictos con los padres y satisfacción con la vida de familia en adolescentes bachilleres. Acta Colombiana de Psicología, 15(1), 77-85. Recuperado de http://sitios.dif.gob.mx/cenddif/wp-cont ent/Archivos/BibliotecaDigital/Funcionam ientoFamiliar.pdf

Luna, A. C. A., \& Laca, F. V. (2014). Análisis factorial confirmatorio de la Escala de Satisfacción con la Vida de Familia (ESFV) en adolescentes de secundaria y bachillerato. Psicogente, 17(31), 226-240. Recuperado de http://www.scielo.org.co/pd f/psico/v17n31/v17n31a16.pdf

Luna, A. C. A., Laca, F. A., \& Mejía, J. C. (2011). Bienestar subjetivo y satisfacción con la vida de familia en adolescentes mexicanos de bachillerato. Psicología Iberoamericana, 19(2), 17-26. Recuperado de http://psycne t.apa.org/record/2012-08454-002

Mardia, K. V. (1970). Measures of multivariate skewness and kurtosis with applications. Biometrika, 57(3), 519-530. https://doi.org/ 10.1093/biomet/57.3.519

McCollum, E. E., Schumm, W. R., \& Russell, C. S. (1988). Reliability and validity of 
the Kansas Family Life Satisfaction Scale in a predominantly middle-aged sample. Psychological Reports, 62(1), 95-98. https://d oi.org/10.2466/pr0.1988.62.1.95

McDonald, R. P. (1999). Test theory: A unified treatment. Mahwah, NJ: Erlbaum.

Merino-Soto, C., \& Salas-Blas, E. (2017). Escala Breve de Búsqueda de Sensaciones (BSSS): estructura latente de las versiones de 8 y 4 ítems en adolescentes peruanos. Adicciones, 30 (1), 41-53. https://doi.org/10.20882/adic ciones. 842

Melton, K. K., Ellis, G., \& Zabriskie, R. (2016). Assessing alternative techniques for scaling the Family Leisure Activity Profile: Recommendations for future family leisure measurement. Leisure Sciences, 38(2), 179-198. https://doi.org/10.1080/01 490400.2015.1087356

Meredith, W., (1993). Measurement invariance, factor analysis and factorial invariance. Psychometrika, 58(4), 525-543. https://doi.o $\mathrm{rg} / 10.1007 / \mathrm{BF} 02294825$

Merino, C., Navarro, J., \& García, W. (2014). Revisión de la consistencia interna del Inventario de Inteligencia Emocional de Bar-On, EQ-I: YV. Revista Peruana de Psicología y Trabajo Social, 3(1), 141-154. ht tps://doi.org/10.13140/2.1.2293.7284

Moss, E., \& Willoughby, B. J. (2016). Associations between beliefs about marriage and life satisfaction: The moderating role of relationship status and gender. Journal of Family Studies, 24(3), 274-290. https://doi.org/10.1080/13229400 .2016 .1187658

Muñiz, J. (1999). Teoría clásica de los tests (2.a ed.). Madrid: Pirámide.

Muñiz, J., \& Hambleton, R. K. (1996). Directrices para la traducción y adaptación de los tests. Papeles del Psicólogo, 66(1), 63-70. Recuperado de http://www.papelesd elpsicologo.es/resumen?pii $=737$

Nunnally, J. C., \& Bernstein, I. J. (1995). Teoría psicométrica (3.ed). México: McGraw-Hill Latinomericana.

Olson, D. H., Bell, R., \& Portner, J. (1978). FACES: Family Adaptability and Cohesion
Evaluation Scales. St. Paul, MN: University of Minnesota.

Olson, D. H., \& Wilson, L. R. (1982). Family Satisfaction Scale. En D. H. Olson, H. I. McCubbin, H. Barnes, A. Larsen, M. Muxen \& M. Wilson (Eds.), Family inventories: Inventories used in a national survey of families across the family life cycle (pp. 43-49). St. Paul, MN: University of Minnesota.

Painuly, N., Sharan, P., \& Mattoo, S. K. (2005). Relationship of anger and anger attacks with depression. European Archives of Psychiatry and Clinical Neuroscience, 255(4), 215-222. https://doi.org/10.1007/s0 0406-004-0539-5

Pérez, E., \& Medrano, L. (2010). Análisis factorial exploratorio: bases conceptuales y metodológicas. Revista Argentina de Ciencias del Comportamiento, 2(1), 58-66. Recuperado de http://www.redalyc.org/htm 1/3334/333427068006/

Poff, R. A., Zabriskie, R. B., \& Townsend, J. A. (2010). Modeling family leisure and related family constructs: A national study of US parent and youth perspectives. Journal of Leisure Research, 42(3), 365-391. Recuperado de https://www.nrpa.org/globalassets/journ als/jlr/2010/volume-42/jlr-volume-42-num ber-3-pp-365-391.pdf

Proctor, C., Linley, P., \& Maltby, J. (2010). Very happy youths: Benefits of very high life satisfaction among adolescents. Social Indicators Research, 98(3), 519-532. https:// doi.org/10.1007/s11205-009-9562-2

Raykov, T., \& Hancock, G. R. (2005). Examining change in maximal reliability for multiplecomponent measuring instruments. British Journal of Mathematical and Statistical Psychology, 58(1), 65-82. https://doi.org/10. 1348/000711005X38753

Rodríguez. M. N., \& Ruiz, M. Á. (2008). Atenuación de la asimetría y de la curtosis de las puntuaciones observadas mediante transformaciones de variables: incidencia sobre la estructura factorial. Psicológica, 29(2), 205-227. Recuperado 
de https://www.uv.es/psicologica/articulos2 .08/6RODRIGUEZ.pdf

Rosenthal, J. A. (1994). Reliability and social work research. Social Work Research, 18(2), 115-121. https://doi.org/10.1093/swr/18.2. 115

Safer, D. J. (2009). Irritable mood and the diagnostic and statistical manual of mental disorders. Child and Adolescent Psychiatry and Mental Health, 3(1), 1-4. https://doi.org /10.1186/1753-2000-3-35

Shek, D., \& Li, X. (2016). Perceived school performance, life satisfaction, and hopelessness: A 4-year longitudinal study of adolescents in Hong Kong. Social Indicators Research, 126(2), 921-934. https://doi.org/1 0.1007/s11205-015-0904-y

Shittu, R. O., Issa, B. A., Olanrewaju, G. T., Mahmoud, A. O., Odeigah, L. O., \& Sule, A. G. (2014). Family dysfunction among depressed HIV/AIDS patients on HAART, in a secondary health institution, in north central Nigeria. HIV 8 AIDS Review, 13(2), 50-55. https://doi.org/10.1016/j.hivar.2014 .02 .005

Schnettler, B., Lobos, G., Miranda-Zapata, E., Denegri, M., Ares, G., \& Hueche, C. (2017b). Diet Quality and Satisfaction with Life, Family Life, and FoodRelated Life across Families: A CrossSectional Pilot Study with Mother-FatherAdolescent Triads. International Journal of Environmental Research and Public Health, 14(11), 1313. https://doi.org/10.3390/ijerp h14111313

Schnettler, B., Miranda-Zapata, E., Grunert, K. G., Lobos, G., Denegri, M., Hueche, C., \& Poblete, H. (2017a). Life satisfaction of university students in relation to family and food in a developing country. Frontiers in Psychology, 8, 15-22. https://doi.org/10.338 9/fpsyg.2017.01522

Schumm, W. R., McCollum, E. E., Bugaighis, M. A., Jurich, A. P., \& Bollman, S. R. (1986). Characteristics of the Kansas Family Life Satisfaction Scale in a regional sample. Psychological Reports, 58(3), 975-980. https: //doi.org/10.2466/pr0.1986.58.3.975
Serrano, M., \& Flores, M. (2004). Relaciones con los padres y apoyo social en adolescentes. Revista de Psicología de la Universidad Autónoma del Estado de México, 15-16, 87-101.

Sheeber, L. B., Davis, B., Leve, C., Hops, H., \& Tildesley, E. (2007). Adolescents' relationships with their mothers and fathers: Associations with depressive disorder and subdiagnostic symptomatology. Journal of Abnormal Psychology, 116(1), 144-154. https://doi.org /10.1037/0021-843X.116.1.144

Smilkstein, G. (1978). The family APGAR: A proposal for a family function test and its uses by physicians. The Journal of Family Practice, 6(6), 1231-1239. Recuperado de https://www.ncbi.nlm.nih.gov/pubmed/ 660126

Stavropoulos, V., Lazaratou, H., Marini, E., \& Dikeos, D. (2015). Low family satisfaction and depression in adolescence: The role of self-esteem. Journal of Educational and Developmental Psychology, 5(2), 109-118. ht tps://doi.org/10.5539/jedp.v5n2p109

Strack, F. (1999). Reports of subjective well-being: Judgmental processes and their methodological implications. En N. Schwarz, D. Kahneman \& E. Diener (Eds.), Well-being: foundations of hedonic psychology (pp. 61-84). Nueva York: Russell Sage Foundation. https://doi.org/10.7758/97816 10443258.8

Suldo, S. M., Huebner, E. S., Friedrich, A. A., \& Gilman, R. (2009). Life satisfaction. En R. Gilman, E. S. Huebner \& M. Furlong (Eds.), Handbook of positive psychology in the schools (pp. 27-35). Nueva York: Routledge.

Suldo, S. M., Savage, J. A., \& Mercer, S. H. (2014). Increasing middle school students' life satisfaction: Efficacy of a positive psychology group intervention. Journal of Happiness Studies, 15(1), 19-42. https://doi. org/10.1007/s10902-013-9414-2

Suldo, S. M., \& Shaffer, E. J. (2008). Looking beyond psychopathology: The dual-factor model of mental health in youth. School Psychology Review, 37(1), 52-68. (N. ${ }^{\circ}$ de 
servicio de reproducción de documentos ERIC EJ817359)

Tabachnick, B. G., Fidell, L. S., \& Osterlind, S. J. (2001). Using multivariate statistics (4.a ed.). Boston: Allyn \& Bacon.

Taşdelen-Karçkay, A. (2016). Family Life Satisfaction Scale-Turkish version: Psychometric evaluation. Social Behavior and Personality, 44(4), 631-640. https://doi. org/10.2224/sbp.2016.44.4.631

Totan, T. t., Özer, A., \& Özmen, O. (2017). The role of hope, life satisfaction, and motivation in bullying among adolescents. International Online Journal of Educational Sciences, 9(2), 391-400. https://doi.org/10.1 5345/iojes.2017.02.008

Ventura-León, J. L. (2017). Intervalos de confianza para coeficiente Omega: propuesta para el cálculo. Adicciones, 30(1), 77-78. https://doi.org/10.20882/adicciones. 962

Ventura-León, J. L., Barboza-Palomino, M., \& Caycho-Rodríguez, T. (2017). iSon necesarios los instrumentos equitativos? Educación Médica, 19(2), 126-127. https://d oi.org/10.1016/j.edumed.2017.03.022

Ventura-León, J. L., \& Caycho-Rodríguez, T. (2017). El coeficiente omega: un método alternativo para la estimación de la confiabilidad. Revista Latinoamericana de Ciencias Sociales, Niñez y Juventud, 15(1), 625-627. Recuperado de http://www.redaly c.org/pdf/773/77349627039.pdf

Widaman, K. F., \& Reise, S. P. (1997). Exploring the measurement invariance of psychological instruments: Applications in the substance use domain. En K. Bryant, M. Windle \& S. West (Eds.), The science of prevention. Methodological advances from alcohol and substance abuse research (pp. 281-324). Washington, DC: American Psychological Association.

Wilson, M. (2005). Constructing measures: An item response modeling approach. Mahwah, NJ: Erlbaum.

Wixom, B. H., \& Todd, P. A. (2005). A theoretical integration of user satisfaction and technology acceptance. Information
Systems Research, 16(1), 85-102. https://doi .org/10.1287/isre.1050.0042

World Health Organization. (1998). The World Health Organisation-Five Well-Being Index [Well-being measures in primary health Care/The DepCare Project]. Copenhagen/ Estocolmo: Autor. Recuperado de https://www.corc.uk.net/outcome-exper ience-measures/the-world-health-organisat ion-five-well-being-index-who-5/

Zabriskie, R., \& McCormick, B. (2003). Parent and child perspectives of family leisure involvement and satisfaction with family life. Journal of Leisure Research, 35(2), 163-189. https://doi.org/10.1080/00222216 .2003 .11949989

Zabriskie, R. B., \& Ward, P. J. (2013). Satisfaction with Family Life Scale. Marriage and Family Review, 49(5), 446-463. https://doi.org/10.1 080/01494929.2013.768321

\section{Notas}

* Artículo de investigación. 